\title{
Current
}

\section{Psychiatric partial hospitalization programs: What you need to know}

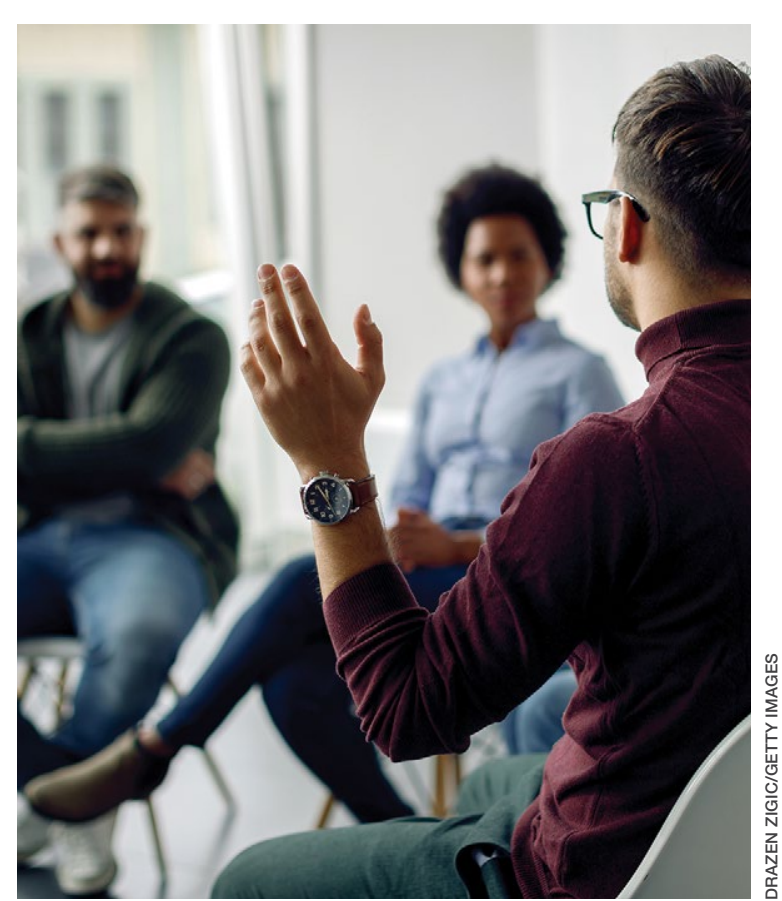

\section{Sudhakar Madakasira, MD, DLFAPA}

President and Chief Medical Director

Psycamore Psychiatric Programs

Flowood, Mississippi

Adjunct Clinical Professor of Psychiatry

University of Mississippi Medical Center

Jackson, Mississippi

\section{PHPs can serve as a useful, cost-effective transition from inpatient to outpatient care}

$\mathrm{P}$

sychiatric partial hospitalization programs (PHPs), previously known as "day hospitals," serve to bridge the gap between inpatient and outpatient facilities by providing intensive, highly structured outpatient behavioral health services (typically several hours of psychotherapy each weekday for most days of the week). The concept of PHPs has existed since at least the 1950s, but such programs started to become more common in the United States as the result of legislation passed in 1963 (Box, ${ }^{1-3}$ page 29). In this article, I provide a brief introductory review of PHPs, while acknowledging that most research on PHPs was conducted years ago and is rather limited.

\section{PHPs: What they are, and how they work}

The term "partial hospitalization" is fraught with confusion because initially it was used to contrast such services from full hospitalization. Historically, it was used to describe services for patients who had been discharged home from a state hospital and attended a program on the hospital grounds during the day as outpatients. In reality, today's PHPs are "day treatment" programs, but the terminology has stuck.

PHPs are neither an inpatient service nor a strict outpatient service, but rather a midground along the continuum of treatment intensity between the 2 traditional types of psychiatric services for patients with a range of mental illness

\section{Disclosure}

The author reports no financial relationships with any companies whose products are mentioned in this article, or with manufacturers of competing products.

doi: 10.12788/cp.0216 
of varying severity. The Association for Ambulatory Behavioral Healthcare, which has set standards and guidelines for PHPs, defines a PHP as "an ambulatory treatment program that includes the major diagnostic, medical, psychiatric, psychosocial, and prevocational treatment modalities designed for patients with serious mental disorders who require coordinated intensive, comprehensive, and multidisciplinary treatment not provided in an outpatient clinical setting." ${ }^{\prime 4}$ PHPs can render acute care as an alternative to inpatient treatment, provide transitional stabilization treatment between an inpatient stay and traditional outpatient treatment (once a week or less frequent), and function as a supplement to traditional outpatient treatment.

Medicare has established criteria that PHPs must meet to qualify for reimbursement ${ }^{5}$; these criteria are now widely accepted as standards of care by the insurance industry. To meet the Medicare criteria, PHP treatment must be active and structured to provide an individualized treatment plan that incorporates coordination of services to meet the particular needs of the patient. ${ }^{5}$ It must include a multidisciplinary team approach to patient care under the direction of a physician, and the treatment goals must be measurable, functional, time-framed, medically necessary, and directly related to the reason for admission. ${ }^{5}$ The physician must certify the medical necessity for admission by documenting that the patient has a diagnosis of an acute Axis I mental disorder, a level of functioning that includes severe impairments in multiple areas of daily life, and a "reasonable expectation" that the disorder and level of functioning will improve as a result of the treatment. ${ }^{5}$

The Joint Commission (formerly JCAHO) lumps day treatment, intensive outpatient, partial hospitalization, and adult day care services into a single category of an ambulatory health care environment offering an organized day or night program of assessment, treatment, care, services, habilitation, or rehabilitation for individuals who do not require 24-hour care. ${ }^{6}$ For behavioral health, this may be a structured, ongoing program that typically meets 2 to 5 times a week for 2 to 5 hours per day. ${ }^{6}$

\section{The growth of partial hospitalization programs}

$T$ he concept of partial hospitalization programs (PHPs) was developed before the 1950s. ${ }^{1}$ However, in the United States, PHPs did not take hold until Congress passed the Community Mental Health Act of 1963, which required that PHPs must be a core component of Community Mental Health Centers (CMHCs). The Omnibus Budget Reconciliation Acts of 1987 and 1990 required Medicare to pay for PHPs affiliated with or based in CMHCs and psychiatric hospitals, which resulted in a proliferation of PHPs across the country. The number of CMHCbased PHPs grew from 296 in 1993 to 769 in $1997 .{ }^{2}$ By 2016 , more than one-third (38.7\%) of all metropolitan hospitals and $11.4 \%$ of nonmetropolitan hospitals in the United States provided PHP services. ${ }^{3}$ This growth was also partially the result of private health insurance companies and the managed care industry clamping down on inpatient hospital stays and approving PHP care to reduce costs. In recent years, freestanding PHPs that are not affiliated with a CMHC or hospital have opened to serve high-functioning patients who do not want inpatient hospitalization or the stigma associated with it.

Most PHPs for adult patients provide services during the day 5 days per week and average 5 to 6 hours of programming per day. Night or evening programming may be a good option for patients who work during the day. Typically, treatment is provided in a group therapy format, with individual therapy at least once a week. Group therapy may include cognitivebehavioral therapy, coping with grief and loss, trauma recovery, conflict resolution, stress management, anger control, and behavioral modification. Family therapy is provided as needed, but usually is mandatory for children and adolescents. Many PHPs offer intensive outpatient programs (IOPs) as a step down to further facilitate a patient's adjustment to psychosocial and family functioning while returning to work on a part-time basis. IOPs typically provide 3 to 4 hours of service per day 3 days per week. While not evidence-based, the typical duration of PHP treatment is 4 to 6 weeks, followed by an additional 2 to 4 weeks of IOP.

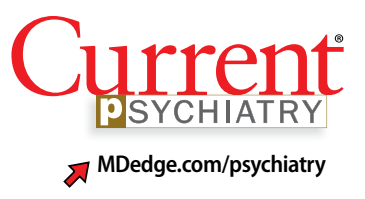

Clinical Point

PHPs typically

provide 5 to 6 hours of group/individual therapy per day, 5 days per week for 4 to 6 weeks

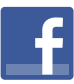

Discuss this article at www.facebook.com/ MDedgePsychiatry 


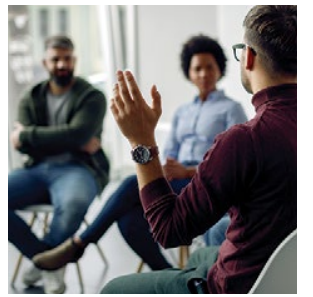

Partial hospitalization

\section{Clinical Point}

One review of 18 studies found that patients and families were more satisfied with PHPs than with inpatient treatment

\section{Partial hospitalization programs: Advantages and disadvantages}

Potential advantages

Discourages the excessive dependency and dehumanization that may develop during inpatient hospitalization

Allows the patient to maintain any independent activities they are capable of despite having a psychiatric disorder

Allows the patient to remain with family

Associated with less stigma

Schedule usually approximates the customary work week, which may make it easier for patients to return to work

Makes it possible to maintain part-time employment Is more demanding and fatiguing for staff or schooling in the evenings and weekends

Offers a longer, more active, and more varied therapeutic experience than inpatient programs

Costs substantially less than inpatient service

Source: Reference 1

\section{Advantages and disadvantages}

Based on a qualitative literature review, Neffinger ${ }^{1}$ outlined potential advantages and disadvantages of PHPs vs inpatient hospitalization $\left(\right.$ Table $\left.^{1}\right)$. While these have not been empirically studied, they may be useful to consider when determining if a PHP would be beneficial for a specific patient.

\section{Which factors are most therapeutic?}

Research on which factors of PHPs are of therapeutic value is quite limited and primarily consists of surveys of small numbers of participants. Hoge et $\mathrm{al}^{7}$ explored the active therapeutic factors responsible for change in the Connecticut Mental Health Center PHP by comparing responses of 20 patients with those of their clinicians. Ninety-five percent of patients rated structure as the top therapeutic factor, followed by interpersonal contact, medication, and altruism. Other factors that were rated as important by $40 \%$ or fewer participants were catharsis, learning, mobilization of family support, connection to community, universality, patient autonomy, successful completion, security, feedback on behavior, and practice at home. In a British study ${ }^{8}$ that

Potential disadvantages

Does not remove the patient from their environment, which for some patients may be desirable or necessary

Is not suitable for patients who may be assaultive or suicidal

May present problems with transportation and/or living arrangements

May interfere with full-time employment or school

Is not suitable for patients with severe cognitive deficits

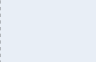

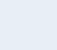


of those who participated in day hospital programs with those of patients who received inpatient care. They found that while overall the 2 approaches produced similar outcomes, there were slightly more positive effects for day hospital programs in measures of symptoms, overall functioning, and social functioning. In terms of cost effectiveness, a 1978 study found that even after correcting for differences in treatment days between inpatient and PHP services, there was a significant financial advantage with PHP (costs were one-third less), primarily because of lower costs per day. ${ }^{12}$ In another study, PHP cost savings were $20 \%$, and potential savings were higher for nonpsychotic patients. ${ }^{13}$

\section{Are PHPs appropriate for children and adolescents?}

Studies of PHPs for adolescents found that patients made gains in peer relationships, behavioral and academic performance, and control of their emotions. ${ }^{14,15}$ A review of PHPs' effectiveness for children suggested that $66 \%$ to $99 \%$ of treated patients demonstrated improvement and successful return to community-based schools, and family functioning was a major factor in improvement. ${ }^{14}$ In a follow-up study that surveyed patients via telephone $>1$ year after discharge from a PHP, almost $80 \%$ of the children and adolescents were either "doing $\mathrm{OK}^{\prime}$ " or were "well-adjusted."14 Only 22.5\% required inpatient or residential treatment; the majority were doing well in school, with only $7 \%$ failing. ${ }^{14}$ In addition, $60 \%$ of parents reported satisfaction with treatment, and $85 \%$ reported functional improvement in their children. ${ }^{14}$

\section{Related Resources}

- Deshmukh P, Kulkarni G, Ganocy SJ. Adults with severe psychiatric symptoms in a community partial hospitalization program: characteristics and predictors of clinical response. Community Ment Health J. 2021;57(4):667-674.

- Khawaja IS. Which patients for partial hospitalization? Current Psychiatry. 2008;7(4):128-128.

\section{Factors that predict PHP success or failure}

In an analysis of a day treatment program that provided 4 months of intensive psychodynamic, group-oriented milieu treatment for patients with long-standing personality disorders, Rosie et $\mathrm{a}^{16}$ found 3 factors that contributed to the success of the PHP:

- optimal treatment-patient matching

- judicious use of authority in milieu therapy

- maintaining close relationships with referral sources.

In a study that compared 58 patients who completed an Ottawa hospital PHP and 44 who did not complete the program, psychological mindedness and chronicity of psychiatric illness were found to predict completion. ${ }^{17}$ However, a study of 59 females with anorexia nervosa who were transferred from inpatient care to an eating disorder day hospital program found that a longer duration of illness, amenorrhea, and a lower body mass index were associated with PHP treatment failure and inpatient rehospitalization. ${ }^{18}$ One study found that for individuals who were referred to a PHP from inpatient care, suicidal ideation and greater psychotic symptoms were associated with acute inpatient rehospitalization. ${ }^{19}$ Other factors associated with PHP nonattendance and treatment failure include limited personal and economic

\section{Bottom Line}

Psychiatric partial hospitalization programs (PHPs) provide a transition from inpatient hospitalization to outpatient treatment for patients who need further stabilization, or serve as an alternative to inpatient treatment for patients who don't need or want inpatient hospitalization. PHPs can be as effective as inpatient treatment for all but the most seriously ill patients, and are more cost-effective than inpatient treatment. 


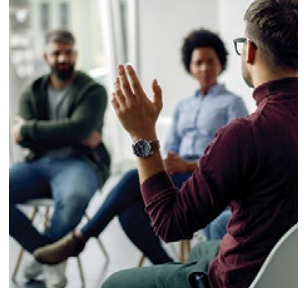

Partial hospitalization

\section{Clinical Point}

Factors associated with PHP treatment failure include high rates of substance abuse disorders, multiple admissions, and disability resources, high rates of substance abuse disorders, multiple admissions, and disability. ${ }^{20}$ In a study of 103 alcohol-dependent patients who completed IOP treatment, 64\% were abstinent at 6 months follow-up; relapse was associated with a longer duration of alcohol dependence and a higher number of prior treatments, while favorable outcomes were associated with a lower degree of depression, anxiety, and craving. ${ }^{21}$ Patients with cocaine dependence who completed an IOP showed significant improvements in addiction scores and had more favorable outcomes in employment status and psychological problems if they stayed longer in treatment. ${ }^{22}$

\section{References}

1. Neffinger GG. Partial hospitalization: an overview. J Community Psychol. 1981;9(3):262-269.

2. Leung MY, Drozd EM, Healy DA, et al. Impacts associated with the Medicare Psychiatric PPS: a study of partial hospitalization programs. February 2009. Accessed January 8, 2022. https://www.cms.gov/Research-Statistics-Dataand-Systems/Statistics-Trends-and-Reports/Reports/ downloads/Leung_PHP_PPS_2010.pdf

3. Williams T, Borders TF, Jasinski L. Partial Psychiatric Hospitalization Program Availability in Non-Metropolitan and Non-Metropolitan Hospitals Nationally. Rural and Underserved Health Research Center; 2019.

4. Rosser J, Michael S, eds. Standards and guidelines for partial hospital programs and intensive outpatient programs. Association for Ambulatory Behavioral Healthcare. 2018. Accessed January 8, 2022. https://aabh.org/wp-content/ uploads/2021/05/2021-SandG-Final.pdf

5. CMS Manual System PUB. 100-02 Medicare Benefit Policy: Partial Hospitalization Services, Department of Health and Human Services. Centers for Medicare \& Medicaid Services; 2004:6-9.

6. Joint Commission. Guide to Joint Commission Behavioral Healthcare Accreditation. Joint Commission; 2007:36.

7. Hoge MA, Farrell SP, Munchel ME, et al. Therapeutic factors in partial hospitalization. Psychiatry. 1988;51(2):199-210.

8. Ricketts T, Kirshbaum MN. Helpfulness of mental health day care: client and staff views. J Adv Nurs. 1994;20(2): 297-306.
9. Marshall M, Crowther R, Almaraz-Serrano A, et al Systematic reviews of the effectiveness of day care for people with severe mental disorders: (1) acute day hospital versus admission; (2) vocational rehabilitation; (3) day hospital versus outpatient care. Health Technol Assess. 2001;5(21):1-75.

10. Horvitz-Lennon M, Normand SL, Gaccione P, et al. Partial versus full hospitalization for adults in psychiatric distress: a systematic review of the published literature (1957-1997). Am J Psychiatry. 2001;158(5):676-685.

11. Sledge WH, Tebes J, Rakfeldt J, et al. Day hospital/crisis respite care versus inpatient care, Part I: Clinical outcomes. Am J Psychiatry. 1996;153(8):1065-1073.

12. Fink EB, Longabaugh R, Stout $R$. The paradoxical underutilization of partial hospitalization. Am J Psychiatry. 1978;135(6):713-716

13. Sledge WH, Tebes J, Wolff N, et al. Day hospital/ crisis respite care versus inpatient care, Part II: service utilization and costs. Am J Psychiatry. 1996;153(8): 1074-1083.

14. Kiser LJ. Treatment-effectiveness research in child and adolescent partial hospitalization. Psychiatr Hosp. 1991; 22(2):51-8.

15. Milin R, Coupland K, Walker S, et al. Outcome and followup study of an adolescent psychiatric day treatment school program. J Am Acad Child Adolesc Psychiatry. 2000;39(3):320-328

16. Rosie JS, Azim HF, Piper WE, et al. Effective psychiatric day treatment: historical lessons. Psychiatr Serv. 1995; 46(10):1019-1026.

17. Tasca GA, Balfour L, Bissada $\mathrm{H}$, et al. Treatment completion and outcome in a partial hospitalization program: interactions among patient variables. Psychotherapy Res. 1999;9(2):232-247.

18. Howard WT, Evans KK, Quintero-Howard CV, et al Predictors of success or failure of transition to day hospital treatment for inpatients with anorexia nervosa. Am J Psychiatry. 1999;156(11):1697-1702.

19. Beard C, Hearon BA, Lee J, et al. When partial hospitalization fails: risk factors for inpatient hospitalization. J Nerv Ment Dis. 2016;204(6):431-436.

20. Lieberman PB, Guggenheim FG. Reasons for patient nonattendance during acute partial hospitalization. Psychiatr Serv. 2016;67(6):684-687.

21. Bottlender M, Soyka M. Efficacy of an intensive outpatient rehabilitation program in alcoholism: predictors of outcome 6 months after treatment. Eur Addict Res. 2005;11(3): 132-137.

22. Gottheil E, Weinstein SP, Sterling RC, et al. A randomized controlled study of the effectiveness of intensive outpatient treatment for cocaine dependence. Psychiatr Serv. 1998; 49(6):782-787. 